\title{
On bounds in Poisson approximation for integer-valued independent random variables
}

\section{Tran Loc Hung* and Le Truong Giang}

\section{"Correspondence:}

thungvn@gmail.com

University of Finance and Marketing,

Ho Chi Minh City, Vietnam

\begin{abstract}
The main aim of this note is to establish some bounds in Poisson approximation for row-wise arrays of independent integer-valued random variables via the Trotter-Renyi distance. Some results related to random sums of independent integer-valued random variables are also investigated.
\end{abstract}

MSC: 60F05; 60G50; 41A36

Keywords: Poisson approximation; random sums; Le Cam's inequality; Trotter's operator; Renyi's operator; probability distance; integer-valued random variable

\section{Introduction}

Let $\left\{X_{n, j}, j=1,2, \ldots, n ; n=1,2, \ldots\right\}$ be a row-wise triangular array of independent integervalued random variables with success probabilities $P\left(X_{n, j}=1\right)=p_{n, j} ; P\left(X_{n, j}=0\right)=1-p_{n, j}-$ $q_{n, j} ; p_{n, j}, q_{n, j} \in(0,1) ; p_{n, j}+q_{n, j} \in(0,1) ; j=1,2, \ldots, n ; n=1,2, \ldots$. Set $S_{n}=\sum_{j=1}^{n} X_{n, j}$ and $\lambda_{n}=$ $E\left(S_{n}\right)=\sum_{j=1}^{n} p_{n, j}$. Suppose that $\lim _{n \rightarrow \infty} \lambda_{n}=\lambda(0<\lambda<+\infty)$. We will denote by $Z_{\lambda}$ the Poisson random variable with mean $\lambda$. It has long been known that in the case of all $q_{n, j}=$ $0(j=1,2, \ldots, n ; n=1,2, \ldots)$, the partial sum $S_{n}$ is said to be a Poisson-binomial random variable, and the probability distributions of $S_{n}, n=1,2, \ldots$, are usually approximated by the distribution of $Z_{\lambda}$. Specially, under the assumptions that $\lim _{n \rightarrow \infty} \max _{1 \leq j \leq n} p_{n, j}=0$, the well-known Poisson approximation theorem states that

$$
S_{n} \stackrel{d}{\rightarrow} Z_{\lambda} \quad \text { as } n \rightarrow \infty .
$$

Here, and from now on, the notation $\stackrel{d}{\rightarrow}$ means the convergence in distribution. It is to be noticed that, for the information on the quality of the Poisson approximation, Le Cam (1960) [1] established the remarkable inequality

$$
\sum_{k=0}^{\infty}\left|P\left(S_{n}=k\right)-P\left(Z_{\lambda}=k\right)\right| \leq 2 \sum_{j=1}^{n} p_{n, j}^{2} .
$$

It is to be noticed that another inequality in Poisson approximation is usually expressed in terms of the total variation distance $d_{T V}\left(S_{n}, Z_{\lambda}\right)$

$$
d_{T V}\left(S_{n}, Z_{\lambda}\right) \leq \sum_{j=1}^{n} p_{n, j}^{2},
$$

๑ 2014 Hung and Giang; licensee Springer. This is an Open Access article distributed under the terms of the Creative Commons Attribution License (http://creativecommons.org/licenses/by/2.0), which permits unrestricted use, distribution, and reproduction in any medium, provided the original work is properly cited. 
where for the distributions $P$ and $Q$ on $\mathbb{Z}_{+}=\{0,1,2, \ldots\}$, the total variation distance between $P$ and $Q$ will be defined as follows:

$$
d_{T V}(P, Q):=\frac{1}{2} \sum_{x \in \mathbb{Z}_{+}}|P(x)-Q(x)| .
$$

(For other surveys, see [1-4], and [5].)

In recent years many powerful tools for establishing the Le Cam's inequality for a wide class of discrete independent random variables have been demonstrated, like the coupling technique, the Stein-Chen method, the semi-group method, the operator method, etc. Results of this nature may be found in [1-11], and [12].

The main aim of this paper is to establish the bounds of the Le Cam-style inequalities for independent discrete integer-valued random variables using the Trotter-Renyi distance based on Trotter-Renyi operator (see [13, 14], for more details). It is to be noticed that during the last several decades the Trotter-operator method has been used in many areas of probability theory and related fields. For a deeper discussion of Trotter's operator we refer the reader to [12-20], and [21].

The results obtained in this paper are extensions of known results in [1, 5, 9-11], and [4]. The present paper is also a continuation of [12].

This paper is organized as follows. The second section deals with the definition and properties of Trotter-Renyi distance, based on Trotter's operator and Renyi's operator. Section 3 gives some results on Le Cam's inequalities, based on the Trotter-Renyi distance, for independent integer-valued distributed random variables. The random versions of these results are also given in this section.

\section{Preliminaries}

In the sequel we shall recall some properties of Trotter-Renyi operator, which has been used for a long time in various studies of classical limit theorems for sums of independent random variables (see [13-15, 18, 19], and [20], for the complete bibliography). Based on Renyi's definition ([14], Chapter 8, Section 12, p.523), we redefine the Trotter-Renyi operator as follows.

Definition 2.1 The operator $A_{X}$ associated with a discrete random variable $X$ is called the Trotter-Renyi operator, defined by

$$
\left(A_{X} f\right)(x)=E(f(X+x))=\sum_{k=0}^{\infty} f(x+k) P(X=k), \quad \forall f \in \mathbb{K}, \forall x \in \mathbb{Z}_{+}
$$

where by $\mathbb{K}$ is denoted the class of all real-valued bounded functions $f$ on the set of all non-negative integers $\mathbb{Z}_{+}:=\{0,1,2, \ldots\}$. The norm of the function $f \in \mathbb{K}$ is defined by $\|f\|=\sup _{x \in \mathbb{Z}_{+}}|f(x)|$.

It is to be noticed that Renyi's operator defined in [14] actually is a discrete form of Trotter's operator (we refer the readers to [13, 15, 17-19], and [20], for a more general and detailed discussion of Trotter's operator).

We shall need in the sequence the following main properties of Trotter-Renyi operator, for all functions $f, g \in \mathbb{K}$ and for $\alpha \in \mathbb{R}$ : 
1. $A_{X}(f+g)=A_{X}(f)+A_{X}(g)$.

2. $A_{X}(\alpha f)=\alpha A_{X}(f)$.

3. $\left\|A_{X}(f)\right\| \leq\|f\|$.

4. $\left\|A_{X}(f)+A_{Y}(f)\right\| \leq\left\|A_{X}(f)\right\|+\left\|A_{Y}(f)\right\|$.

5. Suppose that $A_{X}, A_{Y}$ are operators associated with two independent random variables $X$ and $Y$. Then, for all $f \in \mathbb{K}$,

$$
A_{X+Y}(f)=A_{X} A_{Y}(f)=A_{Y} A_{X}(f) .
$$

In fact, for all $x \in \mathbb{Z}_{+}$

$$
\begin{aligned}
A_{X+Y} f(x) & =\sum_{l=0}^{\infty} f(x+l) P(X+Y=l)=\sum_{r, k=0}^{\infty} f(x+k+r) P(Y=k) P(X=r) \\
& =A_{X}\left(A_{Y} f(x)\right)=A_{X} A_{Y} f(x) .
\end{aligned}
$$

6. Suppose that $A_{X_{1}}, A_{X_{2}}, \ldots, A_{X_{n}}$ are the operators associated with the independent random variables $X_{1}, X_{2}, \ldots, X_{n}$. Then, for all $f \in \mathbb{K}, A_{S_{n}}(f)=A_{X_{1}} A_{X_{2}} \cdots A_{X_{n}}(f)$ is the operator associated with the partial sum $S_{n}=X_{1}+X_{2}+\cdots+X_{n}$.

7. Suppose that $A_{X_{1}}, A_{X_{2}}, \ldots, A_{X_{n}}$ and $A_{Y_{1}}, A_{Y_{2}}, \ldots, A_{Y_{n}}$ are operators associated with independent random variables $X_{1}, X_{2}, \ldots, X_{n}$ and $Y_{1}, Y_{2}, \ldots, Y_{n}$. Moreover, assume that all $X_{i}$ and $Y_{j}$ are independent for $i, j=1,2, \ldots, n$. Then, for every $f \in \mathbb{K}$,

$$
\left\|A_{\sum_{k=1}^{n} X_{k}}(f)-A_{\sum_{k=1}^{n} Y_{k}}(f)\right\| \leq \sum_{k=1}^{n}\left\|A_{X_{k}}(f)-A_{Y_{k}}(f)\right\| .
$$

Clearly,

$$
\begin{aligned}
& A_{X_{1}} A_{X_{2}} \cdots A_{X_{n}}-A_{Y_{1}} A_{Y_{2}} \cdots A_{Y_{n}} \\
& \quad=\sum_{k=1}^{n} A_{X_{1}} A_{X_{2}} \cdots A_{X_{k-1}}\left(A_{X_{k}}-A_{Y_{k}}\right) A_{Y_{k+1}} \cdots A_{Y_{n}} .
\end{aligned}
$$

Accordingly,

$$
\begin{aligned}
\left\|A_{\sum_{k=1}^{n} X_{k}}(f)-A_{\sum_{k=1}^{n} Y_{k}}(f)\right\| & \leq \sum_{k=1}^{n}\left\|A_{X_{1}} \ldots A_{X_{k-1}}\left(A_{X_{k}}-A_{Y_{k}}\right) A_{Y_{k+1}} \cdots A_{Y_{n}}(f)\right\| \\
& \leq \sum_{k=1}^{n}\left\|A_{Y_{k+1}} \cdots A_{Y_{n}}\left(A_{X_{k}}-A_{Y_{k}}\right)(f)\right\| \\
& \leq \sum_{k=1}^{n}\left\|A_{X_{k}}(f)-A_{Y_{k}}(f)\right\| .
\end{aligned}
$$

8. $\left\|A_{X}^{n}(f)-A_{Y}^{n}(f)\right\| \leq n\left\|A_{X}(f)-A_{Y}(f)\right\|$.

Lemma 2.1 The equation $A_{X} f(x)=A_{Y} f(x)$ for $f \in \mathbb{K}, x \in \mathbb{Z}_{+}$shows that $X$ and $Y$ are identically distributed random variables. 
Let $A_{X_{1}}, A_{X_{2}}, \ldots, A_{X_{n}}, \ldots$ be a sequence of Trotter-Renyi's operators associated with the independent discrete random variables $X_{1}, X_{2}, \ldots, X_{n}, \ldots$, and assume that $A_{X}$ is a TrotterRenyi operator associated with the discrete random variable $X$. The following lemma states one of the most important properties of the Trotter-Renyi operator.

Lemma 2.2 A sufficient condition for a sequence of random variables $X_{1}, X_{2}, \ldots, X_{n}, \ldots$ to converge in distribution to a random variable $X$ is that

$$
\lim _{n \rightarrow \infty}\left\|A_{X_{n}}(f)-A_{X}(f)\right\|=0, \quad \text { for all } f \in \mathbb{K} .
$$

Proof Since $\lim _{n \rightarrow \infty}\left\|A_{X_{n}}(f)-A_{X}(f)\right\|=0$, for all $f \in \mathbb{K}$, we conclude that

$$
\lim _{n \rightarrow \infty}\left|\sum_{k=0}^{\infty} f(x+k)\left(P\left(X_{n}=k\right)-P(X=k)\right)\right|=0, \quad \text { for all } f \in \mathbb{K} \text { and for all } x \in \mathbb{Z}_{+} .
$$

Taking

$$
f(x)= \begin{cases}1, & \text { if } 0 \leq x \leq t \\ 0, & \text { if } x>t\end{cases}
$$

then we recover

$$
\lim _{n \rightarrow \infty}\left|\sum_{k=0}^{t}\left(P\left(X_{n}=k\right)-P(X=k)\right)\right|=0 .
$$

It follows that $P\left(X_{n} \leq t\right)-P(X \leq t) \rightarrow 0$ as $n \rightarrow+\infty$. We infer that $X_{n} \stackrel{d}{\rightarrow} X$ as $n \rightarrow+\infty$.

Before stating the definition of the Trotter-Renyi distance we firstly need the definition of a probability metric. Let $(\Omega, \mathbb{A}, \mathbb{P})$ be a probability space and let $\mathbb{Z}(\Omega, \mathbb{A})$ be a space of real-valued $\mathbb{A}$-measurable random variables $X: \Omega \rightarrow \mathbb{R}$.

Definition 2.2 A functional $d(X, Y): \mathbb{Z}(\Omega, \mathbb{A}) \times \mathbb{Z}(\Omega, \mathbb{A}) \rightarrow[0, \infty)$ is said to be a probability metric in $\mathbb{Z}(\Omega, \mathbb{A})$ if it possesses for the random variables $X, Y, Z \in \mathbb{Z}(\Omega, \mathbb{A})$ the following properties (see [2,22] and [18] for more details):

1. $P(X=Y)=1 \Rightarrow d(X, Y)=0$;

2. $\quad d(X, Y)=d(Y, X)$;

3. $d(X, Y) \leq d(X, Z)+d(Z, Y)$.

We now return to the definition of a probability distance based on the Trotter-Renyi operator (see [18, 19], and [21]).

Definition 2.3 The Trotter-Renyi distance $d_{T R}(X, Y ; f)$ of two random variables $X$ and $Y$ with respect to the function $f \in \mathbb{K}$ is defined by

$$
d_{T R}(X, Y ; f):=\left\|A_{X} f-A_{Y} f\right\|=\sup _{x \in \mathbb{Z}_{+}}|E f(X+x)-E f(Y+x)| .
$$


Based on the properties of the Trotter-Renyi operator, some properties of the TrotterRenyi distance are summarized in the following (see [13, 14, 18, 19], and [21] for more details) and we shall omit the proofs.

1. It is easy to see that $d_{T R}(X, Y ; f)$ is a probability metric, i.e. for the random variables $X, Y$, and $Z$ the following properties are possessed:

(a) For every $f \in \mathbb{K}$, the distance $d_{T R}(X, Y$; $f)=0$ if $P(X=Y)=1$.

(b) $d_{T R}(X, Y ; f)=d_{T R}(Y, X ; f)$ for every $f \in \mathbb{K}$.

(c) $d_{T R}(X, Y ; f) \leq d_{T R}(X, Z ; f)+d_{T R}(Z, Y ; f)$ for every $f \in \mathbb{K}$.

2. If $d_{T R}(X, Y ; f)=0$ for every $f \in \mathbb{K}$, then $F_{X} \equiv F_{Y}$.

3. Let $\left\{X_{n}, n \geq 1\right\}$ be a sequence of random variables and let $X$ be a random variable.

The condition

$$
\lim _{n \rightarrow+\infty} d_{T R}\left(X_{n}, X ; f\right)=0, \quad \text { for all } f \in \mathbb{K},
$$

implies that $X_{n} \stackrel{d}{\rightarrow} X$ as $n \rightarrow \infty$.

4. Suppose that $X_{1}, X_{2}, \ldots, X_{n} ; Y_{1}, Y_{2}, \ldots, Y_{n}$ are independent random variables (in each group). Then, for every $f \in \mathbb{K}$,

$$
d_{T R}\left(\sum_{j=1}^{n} X_{j}, \sum_{j=1}^{n} Y_{j} ; f\right) \leq \sum_{j=1}^{n} d_{T R}\left(X_{j}, Y_{j} ; f\right) .
$$

Moreover, if the random variables are identically (in each group), then we have

$$
d_{T R}\left(\sum_{j=1}^{n} X_{j}, \sum_{j=1}^{n} Y_{j} ; f\right) \leq n d_{T R}\left(X_{1}, Y_{1} ; f\right) .
$$

5. Suppose that $X_{1}, X_{2}, \ldots, X_{n} ; Y_{1}, Y_{2}, \ldots, Y_{n}$ are independent random variables (in each group). Let $\left\{N_{n}, n \geq 1\right\}$ be a sequence of positive integer-valued random variables that are independent of $X_{1}, X_{2}, \ldots, X_{n}$ and $Y_{1}, Y_{2}, \ldots, Y_{n}$. Then, for every $f \in \mathbb{K}$,

$$
d_{T R}\left(\sum_{j=1}^{N_{n}} X_{j}, \sum_{j=1}^{N_{n}} Y_{j} ; f\right) \leq \sum_{k=1}^{\infty} P\left(N_{n}=k\right) \sum_{j=1}^{k} d_{T R}\left(X_{j}, Y_{j} ; f\right) .
$$

6. Suppose that $X_{1}, X_{2}, \ldots, X_{n} ; Y_{1}, Y_{2}, \ldots, Y_{n}$ are independent identically distributed random variables (in each group). Let $\left\{N_{n}, n \geq 1\right\}$ be a sequence of positive integer-valued random variables that are independent of $X_{1}, X_{2}, \ldots, X_{n}$ and $Y_{1}, Y_{2}, \ldots, Y_{n}$. Moreover, suppose that $E\left(N_{n}\right)<+\infty, n \geq 1$. Then, for every $f \in \mathbb{K}$, we have

$$
d_{T R}\left(\sum_{j=1}^{N_{n}} X_{j}, \sum_{j=1}^{N_{n}} Y_{j} ; f\right) \leq E\left(N_{n}\right) \cdot d_{T R}\left(X_{1}, Y_{1} ; f\right) .
$$

Finally, we emphasize that the Trotter-Renyi distance in (7) and the total variation distance in (4) have a close relationship if the function $\mathrm{f}$ is chosen as an indicator function of 
a set $A \in \mathbb{Z}_{+}$, namely

$$
f(x)=\chi_{A}(x)= \begin{cases}1, & \text { if } x \in A \\ 0, & \text { if } x \notin A\end{cases}
$$

Then

$$
d_{T R}\left(X, Y, \chi_{A}\right)=d_{T V}(X, Y)
$$

where we denote by $d_{T V}(X, Y)$ the total variation distance between two integer-valued random variables $X$ and $Y$, defined as follows:

$$
d_{T V}(X, Y)=\sup _{A \subseteq \mathbb{Z}_{+}}|P(X \in A)-P(Y \in A)|=\frac{1}{2} \sum_{k \in \mathbb{Z}_{+}}|P(X=k)-P(Y=k)| .
$$

For a deeper discussion of the total variation distance, we refer the reader to [1-4], and [5].

\section{Main results}

Let $\left\{A_{X_{n, j}}, j=1,2, \ldots, n ; n=1,2, \ldots\right\}$ be a sequence of operators associated with the integervalued random variables $X_{n, j}, j=1,2, \ldots, n ; n=1,2, \ldots$, and let $\left\{A_{Z_{p_{n, j}}}, j=1,2, \ldots, n ; n=\right.$ $1,2, \ldots\}$ be a sequence of operators associated with the Poisson random variables with parameters $p_{n, j}, j=1,2, \ldots, n ; n=1,2, \ldots$. Since $Z_{\lambda_{n}}$ is a Poisson random variable with positive parameter $\lambda_{n}=\sum_{j=1}^{n} p_{n, j}$, we can write $Z_{\lambda_{n}} \stackrel{d}{=} \sum_{j=1}^{n} Z_{p_{n, j}}$, where $Z_{p_{n, 1}}, Z_{p_{n, 2}}, \ldots, Z_{p_{n, n}}$ are independent Poisson random variables with positive parameters $p_{n, 1}, p_{n, 2}, \ldots, p_{n, n}$, and the notation $\stackrel{d}{=}$ denotes coincidence of distributions.

Theorem 3.1 Let $\left\{X_{n, j}, j=1,2, \ldots, n ; n=1,2, \ldots\right\}$ be a row-wise triangular array of independent, integer-valued random variables with probabilities $P\left(X_{n, j}=1\right)=p_{n, j}, P\left(X_{n, j}=\right.$ $0)=1-p_{n, j}-q_{n, j} ; p_{n, j}, q_{n, j} \in(0,1) ; p_{n, j}+q_{n, j} \in(0,1) ; j=1,2, \ldots, n ; n=1,2, \ldots$ Let us write $S_{n}=\sum_{j=1}^{n} X_{n, j}$ and $\lambda_{n}=\sum_{j=1}^{n} p_{n, j}$. We will denote by $Z_{\lambda_{n}}$ the Poisson random variable with parameter $\lambda_{n}$. Then, for all functions $f \in \mathbb{K}$,

$$
d_{T R}\left(S_{n}, Z_{\lambda_{n}} ; f\right) \leq 2\|f\| \sum_{j=1}^{n}\left(p_{n, j}^{2}+q_{n, j}\right) .
$$

Proof Applying (8), we have

$$
d_{T R}\left(S_{n}, Z_{\lambda_{n}}, f\right) \leq \sum_{j=1}^{n} d_{T R}\left(X_{n, j}, Z_{p_{n, j}} ; f\right)=\sum_{k=1}^{n}\left\|A_{X_{n, j}}(f)-A_{Z_{p_{n, j}}}(f)\right\| .
$$

Moreover, for all $f \in \mathbb{K}$, for all $x \in \mathbb{Z}_{+}$and $r \in\{0,1, \ldots, n\}$ we conclude that

$$
\begin{aligned}
A_{X_{n j}} f(x)-A_{Z_{p_{n, j}}} f(x) & =\sum_{r=0}^{\infty} f(x+r)\left(P\left(X_{n j}=r\right)-P\left(Z_{\lambda_{p, j}}=r\right)\right) \\
& =\sum_{r=0}^{\infty} f(x+r)\left(P\left(X_{n j}=r\right)-\frac{e^{-p_{n, j}} p_{n, j}^{r}}{r !}\right)
\end{aligned}
$$




$$
\begin{aligned}
= & f(x)\left(1-p_{n, j}-q_{n, j}-e^{-p_{n, j}}\right) \\
& +f(x+1)\left(p_{n, j}-p_{n, j} e^{-p_{n, j}}\right) \\
& +\sum_{r=2}^{\infty} f(x+r)\left(P\left(X_{n, j}=r\right)-\frac{e^{-p_{n, j}} p_{n, j}^{r}}{r !}\right) .
\end{aligned}
$$

Therefore, for all functions $f \in K$, and for all $x \in \mathbb{Z}_{+}$, we have

$$
\begin{aligned}
\left|A_{X_{n, j}} f(x)-A_{Z_{p_{n, j}}} f(x)\right| & \mid f(x)\left(1-p_{n, j}-q_{n, j}-e^{-p_{n, j}}\right)+f(x+1)\left(p_{n, j}-p_{n, j} e^{-p_{n, j}}\right) \\
& +\sum_{r=2}^{\infty} f(x+r)\left(P\left(X_{n, j}=r\right)-\frac{e^{-p_{n, j}} p_{n, j}^{r}}{r !}\right) \mid \\
= & \left|f(x)\left(1-p_{n, j}-q_{n, j}-e^{-p_{n, j}}\right)\right|+\left|f(x+1)\left(p_{n, j}-p_{n, j} e^{-p_{n, j}}\right)\right| \\
& +\left|\sum_{r=2}^{\infty} f(x+r)\left(P\left(X_{n, j}=r\right)-\frac{e^{-p_{n, j}} p_{n, j}^{r}}{r !}\right)\right| \\
\leq & \left|f(x)\left(1-p_{n, j}-q_{n, j}-e^{-p_{n, j}}\right)\right|+\left|f(x+1)\left(p_{n, j}-p_{n, j} e^{-p_{n, j}}\right)\right| \\
& +\left|\sum_{r=2}^{\infty} f(x+r) P\left(X_{n, j}=r\right)\right|+\left|\sum_{r=2}^{\infty} f(x+r) \frac{e^{-p_{n, j}} p_{n, j}^{r}}{r !}\right| \\
\leq & \left(e^{-p_{n, j}}+p_{n, j}+q_{n, j}-1\right) \sup _{x \in \mathbb{Z}_{+}}|f(x)|+\left(p_{n, j}-p_{n, j} e^{-p_{n, j}}\right) \sup _{x \in \mathbb{Z}_{+}}|f(x)| \\
& +\sup _{x \in \mathbb{Z}_{+}}|f(x)|\left|\sum_{r=2}^{\infty} P\left(X_{n, k}=r\right)\right|+\sup _{x \in \mathbb{Z}_{+}}|f(x)|\left|\sum_{r=2}^{\infty} \frac{e^{-p_{n, j}} p_{n, j}^{r}}{r !}\right| \\
= & \sup _{x \in \mathbb{Z}_{+}}|f(x)|\left(e^{-p_{n, j}}+p_{n, j}+q_{n, j}-1+p_{n, j}-p_{n, j} e^{-p_{n, j}}+q_{n, j}+1-e^{-p_{n, j}}-p_{n, j} e^{-p_{n, j}}\right) \\
= & 2\|f\|\left(p_{n, j}-p_{n, j} e^{-p_{n, j}}+q_{n, j}\right) \\
\leq & 2\|f\|\left(p_{n, j}^{2}+q_{n, j}\right) . \\
= & \\
= &
\end{aligned}
$$

One infers that

$$
\forall f \in K, \quad\left\|A_{X_{n, j}}(f)-A_{Z_{p_{n, j}}}(f)\right\| \leq 2\|f\|\left(p_{n, j}^{2}+q_{n, j}\right) .
$$

Therefore, applying (8), we can assert that

$$
d_{T R}\left(S_{n}, Z_{\lambda_{n}} ; f\right) \leq 2\|f\| \sum_{j=1}^{n}\left(p_{n, j}^{2}+q_{n, j}\right) .
$$

This completes the proof.

Corollary 3.1 Under the assumptions of Theorem 3.1 , let $r \in\{0,1, \ldots, n\}$, we have

$$
\left|P\left(S_{n}=r\right)-P\left(Z_{\lambda_{n}}=r\right)\right| \leq 2 \sum_{j=1}^{n}\left(p_{n, j}^{2}+q_{n, k}\right) .
$$


Remark 3.1 We consider Corollary 3.1 and assume that the following conditions are satisfied:

$$
\begin{aligned}
& \text { (i) } \lim _{n \rightarrow \infty} \sum_{j=1}^{n} q_{n, j}=0, \\
& \text { (ii) } \lim _{n \rightarrow \infty} \max _{1 \leq k \leq n} p_{n, j}=0, \\
& \text { (iii) } \lim _{n \rightarrow \infty} \lambda_{n}=\lim _{n \rightarrow \infty} \sum_{j=1}^{n} p_{n, j}=\lambda \quad(0<\lambda<+\infty) .
\end{aligned}
$$

Then $S_{n} \stackrel{d}{\rightarrow} Z_{\lambda}$ as $n \rightarrow \infty$.

Theorem 3.2 Let $\left\{X_{n, j}, j=1,2, \ldots, n ; n=1,2, \ldots\right\}$ be a row-wise triangular array of independent, integer-valued random variables with probabilities $P\left(X_{n, j}=1\right)=p_{n, j}, P\left(X_{n, j}=0\right)=$ $1-p_{n, j}-q_{n, j} ; p_{n, j}, q_{n, j} \in(0,1) ; p_{n, j}+q_{n, j} \in(0,1) ; j=1,2, \ldots, n ; n=1,2, \ldots$ Moreover, we suppose that $N_{n}, n=1,2, \ldots$ are positive integer-valued random variables, independent of all $X_{n, j}, j=1,2, \ldots, n ; n=1,2, \ldots$. Let us write $S_{N_{n}}=\sum_{j=1}^{N_{n}} X_{n, j}$ and $\lambda_{N_{n}}=\sum_{j=1}^{N_{n}} p_{n, j}$. We will denote by $Z_{\lambda_{N_{n}}}$ the Poisson random variable with parameter $\lambda_{N_{n}}$. Then, for all functions $f \in \mathbb{K}$,

$$
d_{T R}\left(S_{N_{n}}, Z_{\lambda_{N_{n}}} ; f\right) \leq 2\|f\| E\left(\sum_{j=1}^{N_{n}}\left(p_{N_{n}, j}^{2}+q_{N_{n}, j}\right)\right) .
$$

Proof According to Theorem 3.1 and (9), for all functions $f \in \mathbb{K}$, and for all $x \in \mathbb{Z}_{+}$, we have

$$
\begin{aligned}
d_{T R}\left(S_{N_{n}}, Z_{\lambda_{N_{n}}} ; f\right) & \leq \sum_{m=1}^{\infty} P\left(N_{n}=m\right) d_{T R}\left(S_{m}, Z_{\lambda_{m}} ; f\right) \\
& \leq \sum_{m=1}^{\infty} P\left(N_{n}=m\right) 2\|f\| \sum_{j=1}^{m}\left(p_{N_{n}, j}^{2}+q_{N_{n}, j}\right) \\
& =2\|f\| \sum_{m=1}^{\infty}\left[P\left(N_{n}=m\right) \sum_{j=1}^{m}\left(p_{N_{n}, j}^{2}+q_{N_{n}, j}\right)\right] \\
& =2\|f\| E\left(\sum_{j=1}^{N_{n}}\left(p_{N_{n}, j}^{2}+q_{N_{n}, j}\right)\right) .
\end{aligned}
$$

Therefore,

$$
d_{T R}\left(S_{N_{n}}, Z_{\lambda_{N_{n}}} ; f\right) \leq 2\|f\| E\left(\sum_{j=1}^{N_{n}}\left(p_{N_{n}, j}^{2}+q_{N_{n}, j}\right)\right) .
$$

The proof is complete.

Corollary 3.2 According to Theorem 3.2, let $r \in\{0,1, \ldots, n\}$, we have

$$
\left|P\left(S_{N_{n}}=r\right)-P\left(Z_{\lambda_{N_{n}}}=r\right)\right| \leq 2 E\left(\sum_{j=1}^{N_{n}}\left(p_{N_{n}, j}^{2}+q_{N_{n}, j}\right)\right) .
$$


Theorem 3.3 Let $\left\{X_{k, j}\right\}(k=1,2, \ldots ; j=1,2, \ldots)$ be a double array of independent integervalued random variables with probabilities $P\left(X_{k, j}=1\right)=p_{k, j}, P\left(X_{k, j}=0\right)=1-p_{k, j}-q_{k, j}$, $p_{n, k} \in(0,1) ; k=1,2, \ldots ; j=1,2, \ldots$ Assume that for every $k=1,2, \ldots$ the random variables $X_{k, 1}, X_{k, 2}, \ldots$, are independent, and for every $j=1,2, \ldots$ the random variables $X_{1, j}, X_{2, j}, \ldots$ are independent. Set $S_{n m}=\sum_{k=1}^{n} \sum_{j=1}^{m} X_{k, j}$. Let us denote by $Z_{\delta_{n, m}}$ the Poisson random variable with mean $\delta_{n, m}=\sum_{k=1}^{n} \sum_{j=1}^{m} p_{k, j}$. Then, for all $f \in \mathbb{K}$,

$$
d_{T R}\left(S_{n m}, Z_{\delta_{n, m}}, f\right) \leq 2\|f\| \sum_{k=1}^{n} \sum_{j=1}^{m}\left(p_{k, j}^{2}+q_{k, j}\right)
$$

Proof Applying the inequality in (8), we have

$$
\begin{aligned}
d_{T R}\left(S_{n m}, Z_{\delta_{n m}}, f\right) & \leq \sum_{k=1}^{n} d_{T R}\left(S_{k m}, Z_{\mu_{k, m}}, f\right) \\
& \leq \sum_{k=1}^{n} \sum_{j=1}^{m} d_{T R}\left(S_{k, j}, Z_{\lambda_{k, j}}, f\right) .
\end{aligned}
$$

According to Theorem 3.1, for all functions $f \in \mathbb{K}$, and for all $x \in \mathbb{Z}_{+}$, we conclude that

$$
d_{T R}\left(S_{k, j}, Z_{\lambda_{k, j}} f\right) \leq 2\|f\|\left(p_{k, j}^{2}+q_{k, j}\right) .
$$

Therefore,

$$
d_{T R}\left(S_{n m}, Z_{\delta_{n m}}, f\right) \leq 2\|f\| \sum_{k=1}^{n} \sum_{j=1}^{m}\left(p_{k, j}^{2}+q_{k, j}\right) .
$$

This completes the proof.

Theorem 3.4 Let $\left\{X_{k, j}, k=1,2, \ldots ; j=1,2, \ldots\right\}$ be a double array of independent integervalued random variables with $P\left(X_{k, j}=1\right)=p_{k, j} ; P\left(X_{k, j}=0\right)=1-p_{k, j}-q_{k, j} ; p_{k, j}, q_{k, j} \in(0,1)$; $p_{k, j}+q_{k, j} \in(0,1) ; k=1,2, \ldots ; n=1,2, \ldots$. Assume that for every $k=1,2, \ldots$ the random variables $X_{k, 1}, X_{k, 2}, \ldots$, are independent, and for every $j=1,2, \ldots$ the random variables $X_{1, j}, X_{2, j}, \ldots$ are independent. Set $S_{n m}=\sum_{k=1}^{n} \sum_{j=1}^{m} X_{k, j}$. Suppose that $N_{n}, M_{m}$ are non-negative integer-valued random variables independent of all $X_{n, m}, n \geq 1 ; m \geq 1$. Let us denote by $Z_{\delta_{N_{n} M_{m}}}$ the Poisson random variable with mean $\delta_{N_{n} M_{m}}=E\left(S_{N_{n} M_{m}}\right)=$ $\sum_{k=1}^{N_{n}} \sum_{j=1}^{M_{m}} p_{k, j}$. Then, for all functions $f \in \mathbb{K}$,

$$
d_{T R}\left(S_{N_{n} M_{m}}, Z_{\delta_{N_{n} M_{m}}}, f\right) \leq 2\|f\| E\left(\sum_{k=1}^{N_{n}} \sum_{j=1}^{M_{n}}\left(p_{k, j}^{2}+q_{k, j}\right)\right) .
$$

Proof According to Definition 2.1, we have

$$
\begin{aligned}
\left(A_{S_{N_{n} M_{m}}} f\right)(x) & :=E\left(f\left(S_{N_{n} M_{m}}+x\right)\right) \\
& =\sum_{n=1}^{\infty} P\left(N_{n}=n\right) \sum_{m=1}^{\infty} P\left(M_{n}=m\right)\left(A_{S_{n m}} f\right)(x)
\end{aligned}
$$


and

$$
\begin{aligned}
\left(A_{Z_{\delta_{N_{n} M} M}} f\right)(x): & =E\left(f\left(Z_{\delta_{N_{n} M_{m}}}+x\right)\right) \\
& =\sum_{n=1}^{\infty} P\left(N_{n}=n\right) \sum_{m=1}^{\infty} P\left(M_{n}=m\right)\left(A_{Z_{\delta_{n m}}} f\right)(x) .
\end{aligned}
$$

Therefore, for all functions $f \in \mathbb{K}$, and for all $x \in \mathbb{Z}_{+}$, we have

$$
\begin{aligned}
& \left\|A_{S_{N_{n} M_{m}}}(f)-A_{Z_{\delta_{N_{n} M_{m}}}}(f)\right\| \\
& \leq \sum_{n=1}^{\infty} P\left(N_{n}=n\right) \sum_{m=1}^{\infty} P\left(M_{n}=m\right)\left\|A_{S_{n m}}(f)-A_{Z_{\delta_{n, m}}}(f)\right\| \\
& \leq 2\|f\| \sum_{n=1}^{\infty} P\left(N_{n}=n\right) \sum_{m=1}^{\infty} P\left(M_{n}=m\right)\left(\sum_{k=1}^{n} \sum_{j=1}^{m}\left(p_{k, j}^{2}+q_{k, j}\right)\right) \\
& =2\|f\| \sum_{n=1}^{\infty} P\left(N_{n}=n\right) E\left(\sum_{k=1}^{n} \sum_{j=1}^{M_{m}}\left(p_{k, j}^{2}+q_{k, j}\right)\right) \\
& =2\|f\| E\left(\sum_{k=1}^{N_{n}} \sum_{j=1}^{M_{m}}\left(p_{k, j}^{2}+q_{k, j}\right)\right) .
\end{aligned}
$$

Thus,

$$
d_{T R}\left(S_{N_{n} M_{m}}, Z_{\delta_{N_{n}, M_{m}}}, f\right) \leq 2\|f\| E\left(\sum_{k=1}^{N_{n}} \sum_{j=1}^{M_{n}}\left(p_{k, j}^{2}+q_{k, j}\right)\right) .
$$

The proof is straightforward.

Remark 3.2 In the case of all probabilities $q_{n, j}=0, j=1,2, \ldots, n ; n=1,2, \ldots$ the partial sum $S_{n}=\sum_{j=1}^{n} X_{n, j}$ will become a Poisson-binomial random variable, and one concludes that the results of Theorems 3.1, 3.2, 3.3, and 3.4 are extensions of results in [12] (see [12] for more details).

We conclude this paper with the following comments. The Trotter-Renyi distance method is based on the Trotter-Renyi operator and it has a big application in the Poisson approximation. Using this method it is possible to establish some bounds in the Poisson approximation for sums (or random sums) of independent integer-valued random vectors.

\section{Competing interests}

The authors declare that they have no competing interests.

\section{Authors' contributions}

All authors contributed equally and significantly to this work. All authors drafted the manuscript, read and approved the final version of the manuscript.

\section{Acknowledgements}

The authors wish to express their gratitude to the referees for valuable remarks and comments, improving the previous version of this paper. The research was supported by the Vietnam National Foundation for Science and Technology Development (NAFOSTED, Vietnam) under grant 101.01-2010.02. 


\section{References}

1. Le Cam, L: An approximation theorem for the Poisson binomial distribution. Pac. J. Math. 10(4), 1181-1197 (1960)

2. Barbour, AD, Holst, L, Janson, S: Poisson Approximation. Clarendon, Oxford (1992)

3. Chen, LHY, Leung, D: An Introduction to Stein's Method. Singapore University Press, Singapore (2004)

4. Neammanee, KA: Nonuniform bound for the approximation of Poisson binomial by Poisson distribution. Int. J. Math. Math. Sci. 48, 3041-3046 (2003)

5. Steele, JM: Le Cam's inequality and Poisson approximations. Am. Math. Mon. 101(1), 48-50 (1994)

6. Arratia, R, Goldstein, L, Gordon, L: Poisson approximation and the Chen-Stein method. Stat. Sci. 5, 403-434 (1990)

7. Chen, LHY: On the convergence of Poisson binomial to Poisson distribution. Ann. Probab. 2(1), 178-180 (1974)

8. Deheuvels, P, Karr, A, Pfeifer, D, Serfling, R: Poisson approximations in selected metrics by coupling and semigroup methods with applications. J. Stat. Plan. Inference 20, 1-22 (1988)

9. Teerapabolarn, K, Wongkasem, P: Poisson approximation for independent geometric random variables. Int. Math. Forum 2, 3211-3218 (2007)

10. Teerapabolarn, K: A note on Poisson approximation for independent geometric random variables. Int. Math. Forum 4 , 531-535 (2009)

11. Teerapabolarn, K: A pointwise approximation for independent geometric random variables. Int. J. Pure Appl. Math. 76, 727-732 (2012)

12. Hung, $T L$, Thao, $V T$ : Bounds for the approximation of Poisson-binomial distribution by Poisson distribution. J. Inequal. Appl. 2013, 30 (2013)

13. Trotter, HF: An elementary proof of the central limit theorem. Arch. Math. (Basel) 10, 226-234 (1959)

14. Renyi, A: Probability Theory. North-Holland, Amsterdam (1970)

15. Butzer, PL, Hahn, L, Westphal, U: On the rate of approximation in the central limit theorem. J. Approx. Theory 13 327-340 (1975)

16. Rychlick, R, Szynal, D: On the rate of convergence in the central limit theorem. In: Probability Theory, vol. 5, pp. 221-229. Banach Center Publications, Warsaw (1979)

17. Cioczek, R, Szynal, D: On the convergence rate in terms of the Trotter operator in the central limit theorem without moment conditions. Bull. Pol. Acad. Sci., Math. 35(9-10), 617-627 (1987)

18. Kirschfink, H: The generalized Trotter operator and weak convergence of dependent random variables in different probability metrics. Results Math. 15, 294-323 (1989)

19. Hung, TL: On a probability metric based on Trotter operator. Vietnam J. Math. 35(1), 22-33 (2007)

20. Hung, TL: Estimations of the Trotter's distance of two weighted random sums of $d$-dimensional independent random variables. Int. Math. Forum 4, 1079-1089 (2009)

21. Hung, TL, Thanh, TT: On the rate of convergence in limit theorems for random sums via Trotter-distance. J. Inequal. Appl. 2013, 404 (2013)

22. Zolotarev, VM: Probability metrics. Theory Probab. Appl. 28, 278-302 (1983)

doi:10.1186/1029-242X-2014-291

Cite this article as: Hung and Giang: On bounds in Poisson approximation for integer-valued independent random variables. Journal of Inequalities and Applications 2014 2014:291.

\section{Submit your manuscript to a SpringerOpen ${ }^{\circ}$ journal and benefit from:}

- Convenient online submission

- Rigorous peer review

- Immediate publication on acceptance

- Open access: articles freely available online

- High visibility within the field

- Retaining the copyright to your article 